\title{
Consideración del factor percepción visual en el material bibliográfico sobre "Movilidad urbana para una planificación sostenible"
}

Sofía Letelier P.

\section{Filiación}

Académica de la F.A.U. de la Universidad de Chile.

\section{Resumen:}

Pesquisa, análisis y reflexión sobre los tópicos manejados en la postulación y discusión acerca de la movilidad urbana sostenible, que aluden o consideran explícita o implícitamente en algún grado la incidencia del factor Percepción Visual. El estudio se circunscribe a los postulados, estrategias, instrumentos, normativas, ejemplos y sugerencias proporcionados como bibliografía básica y complementaria al curso "Movilidad y Planeamiento Sostenible". Para su revisión se elige el enfoque de la Percepción Visual (PV), dada la necesidad que tiene la filosofía de la sostenibilidad de concluir en 'artes' concretas, dispositivos, señaléticas y demás artilugios que deben ser captados a distancia, para finalmente inducir conductas y comportamientos. De no ser así, no se alcanzarían resultados esperados. Este enfoque se aplica, primero, bajo la hipótesis de que la PV, no obstante ser un filtro que se apropia personalizadamente de la realidad, se apoya en mecanismos comunes a la especie pero también culturales que responden y son centralmente activados y condicionados por atributos específicos de los estímulos o por condiciones de su contexto, lo que hace que el fenómeno de la PV en sus rasgos y efectos globales estructurales, pueda ser anticipado o vaticinado. En segundo lugar, suponiendo que la PV, por ser un fenómeno activo - que 'construye la realidad' en el circuito de las significaciones e 'ideas significativas'- es una experiencia que tanto promueve como inhibe conductas y comportamientos determinados. Para el efecto, luego de una puesta en común de los aspectos de la PV que se estiman a priori como incidentes a la escala urbana y a objeto de explicitar el alcance y profundidad que nos ocupa, nos abocamos a constatar la existencia de rasgos inductores, reforzadores o facilitadores de la percepción visual o bien que son disuasivos, distractores, ambiguadores o entorpecedores, perceptuales, deduciendo sus efectos y posibles factores que los explican.

Palabras claves: Percepción visual, movilidad urbana.

\begin{abstract}
This is an investigation, analysis and reflection on the discussion and propositions about sustainable urban mobility, specially, on subjects that imply or concern directly the Visual Perception. This investigation is inscribed within the propositions, strategies, instruments, laws, examples and suggestions given as a bibliography to the course "Mobility and Sustainable Planning" (Eng. Dr. J. Pozueta). A point of view that focuses in Visual Perception to analyze the mobility is used because it is a necessity for the sustainability philosophy to come up with concrete "arts", displays, signals, etc., that must be seen from a distance so that they may persuade a change in conducts and
\end{abstract}


http://revistaurbanismo.uchile.cl

behavior. If these "arts" were not clear in their message and perception the objectives will not be accomplished.

\section{Key words}

Visual perception, sustainable urban mobility.

\section{Sumario}

1. Importancia de la Percepción Visual en la 'Lectura' Urbana y en la Movilidad

2. Presencia del factor Percepción Visual en las publicaciones sobre Movilidad Urbana Sostenible

3. Efectos Perceptuales que incidirían en el éxito de algunas estrategias para una Movilidad Sostenible

4. Conclusiones

\section{1.- Importancia de la Percepción Visual en la 'Lectura' Urbana y en la Movilidad}

\section{1.- El 'Urbanita' y la percepción}

Parece obvio decir que el ser humano crea artificios complejos como las ciudades, entre otras razones, para disfrutarlos; pero para ello requiere que éstos no escapen a su entendimiento, que faciliten su comprensión y orientación, de manera que no se transformen en un arcano amenazante y le permitan el disfrute estético de lo creado.

La Percepción, en tanto mecanismo interno que participa en el proceso de las 'representaciones semánticas' (Per Aage Brandt, 2001), es reactiva ante la realidad al operar activando nociones abstraídas de la propia realidad - en un circuito iterativo- . La percepción funcionaría como un tamiz o filtro (A. Rapoport, 1978) que nos entrega 'perceptos' - unidades de registro + el sentido de las cosas -, los cuales se constituyen en nuestras unidades de conocimiento de la realidad; unidades de experiencia que permiten operar con el entorno. Percibir se transforma entonces un proceso activo que -sostenemos - funcionaría en forma instantánea como una 'inducción completa': depende, por una parte de la deducción que derivamos desde las nociones o ideas significativas que nos hemos formado de la realidad y que nos entrega una primera confianza ("vemos lo que sabemos ver"); y, por otra, de la inducción que, a partir de las características y atributos particulares de lo que se presenta como estímulo a los sentidos y a la consciencia, nos permite construir ese 'percepto' que nos hace sentido y que pasamos a ver como un mundo real.

Tratándose de movilidad en la ciudad y dada su escala, la que necesita manifestarse desde la distancia, entre todos los canales perceptivos es la Percepción Visual la que adquiere la mayor importancia por su propiedad telemétrica. Aún quienes la tienen limitada o son carentes, se ven en la necesidad de acceder de algún modo a los mensajes urbanos, prever situaciones y orientarse. Es también mediante la PV que nos formamos las imágenes - incluso de lo que hemos recorrido y experimentado ocupando 
otros registros - al darnos el sentido de totalidad y las relaciones de organización, permitiéndonos captar las estructuras subyacentes y construir nuestros 'mapas mentales' entre puntos de interés. Pero, más allá de la escala, y muy especialmente por su estereometría que nos entrega la profundidad, la PV nos permite vaticinar el escenario en su espacialidad, distancias y ubicación relativa de las cosas.

\section{2. La Percepción Visual como promotora de comportamiento}

La Percepción Visual (que cuando es suplida por otras formas de registro sensorial, no alcanza a ser nunca totalmente reemplazada) es particularmente incidente en el comportamiento individual y social, dadas sus propiedades vinculativas ( 1 ) que relacionan los componentes de la realidad. Hoy se habla de 'inteligencia visual' refiriéndose a la actividad que acomete la mente al 'leer' la realidad mediante la percepción, capaz de realizar operaciones complejas como son: la selección (que aísla lo que interesa dejando todo el resto como 'fondo'); la clasificación (que ordena, jerarquiza y categoriza); la evaluación (que asigna valor y pondera para actuar); el simbolismo (que significa y abstrae ) y la autoconsciencia del entorno (referencia a uno mismo para la asignación significante - como p.ej. "lejos/cerca"- ).

Esta forma de inteligencia vinculativa se caracterizaría por:

- Ser inmediata

- Ser una forma de experiencia válida ( no sólo lo que se recorre, también lo que se ve)

- Ser objetivable (deja claro lo que me es externo) y, por lo tanto, generalizable.

- Ser móvil, y enseña a suponer variantes (de puntos de vista) sin desorientarnos.

- Ser discriminatoria ( ve lo que quiere, puede o sabe, ver)

- Permite advertir regularidades, deducir constancias y darnos certezas (cabalidad).

- Ser estereométrica, entregándonos la profundidad (consciencia tridimensional y de las distancias) y la orientación.

Estas características son simultáneas e indivisibles en el momento del entendimiento y reacción al ambiente. Pero, siendo las primeras características adjetivas, las tres últimas son activas y dependen directamente de las claves que entrega el estímulo.

Ello es pertinente al planeamiento para una movilidad urbana sostenible, ya que éste propone artificios y situaciones que necesitan ser fácilmente percibidas e internalizadas del modo previsto, a fin de provocar determinada reacción. Pero como nada se percibe aisladamente, las decisiones objetivas materiales y las medidas estratégicas que les conciernen, necesitan considerar estas propiedades vinculativas de la percepción, desde el manejo de sus características, ya que ellas funcionarán quiérase o no.

Adicionalmente, se deberá tener en cuenta que las disposiciones urbanas se dirigen a satisfacer a un universo variado de usuarios que, al menos, tendrá dos modos generales y personales de percibir: un modo analítico (en el que predominan los mecanismos deductivos de lectura), o bien y un modo sintético (dócil a los rasgos capaces de imponerse a la percepción más inductiva). Como ambos modos están 
presentes en todos los individuos, pero éstos poseen distintos contenidos y criterios de análisis, es el último el que interesa activar, dado que es el único que podemos operar. Porque, si bien las respuestas se condicionarán en parte por las características de cada individuo y esa variedad no puede ser controlada, sabemos que sí podremos actuar sobre los atributos de los estímulos que se les presenten, atributos que actuarán, al menos, sobre los mecanismos comunes de especie. De modo que si se desea asegurar una conducta social en algún grado homogénea, sólo se puede apostar a la capacidad de inducción del estímulo y de que éste permita y facilite determinada síntesis. El modo sintético de percibir es fundamental, además, porque cualquier movilización en la ciudad que no sea la peatonal, requiere de una rapidez de reacción que invalida la utilidad del modo analítico de percibir.

\section{3.- Mecanismos que operan propiedades vinculativas en la percepción sintética}

\subsection{1.- Mecanismos situacionales}

Además de las características generales de las propiedades vinculativas de la percepción a que nos hemos referido y que nos permiten leer el entorno como un todo, existen mecanismos perceptivos (2) que responden más directa y precisamente a la situación en que se dan los atributos del estímulo y al entorno que le rodea (importante, porque el contexto de un atributo puede variarlo radicalmente, produciendo equívocos visuales ) atributos y entorno que, en conjunto, entregarán determinada lectura apoyando o inhibiendo la captación del mensaje implícito y, consecuentemente, induciendo los comportamientos derivados. Estos mecanismos son:

- Nivelación / Agudización (se 'aplanan' los atributos o estímulos entre sí y hacen indistinguibles o se resalta uno atributo o estímulo por sobre otro).

- Articulación de campo (distingue partes de un todo; discrimina efectos debidos al campo visual o entorno).

- Alcance (promueve prestar la atención a campos amplios o bien a concentrarla).

Es evidente que evitar los enunciados confusos e indecidibles o ambiguos donde se imponga la nivelación; controlar la captación total del enunciado distinguiendo sus partes y los efectos debidos al campo visual o el soporte; y prever el alcance de la atención en determinada situación, son aspectos que los criterios de diseño deben considerar tomando en cuenta los umbrales de reacción relativos a estos mecanismos, que si bien pueden ser modelados culturalmente, son generales de especie ( $\underline{3}$ ), según las Teorías del 'estado directivo'.

\subsection{2.- Construcción y Experiencia de la 'escala' y de la 'profundidad'}

No obstante la importancia del modo inductivo en la percepción, la capacidad de captar las denominadas 'constancias' del entorno (4), basadas en la experiencia, utiliza el modo deductivo de percibir, haciendo que aquel no parezca una amenaza. Por las constancias sabemos, p.ej. que la carretera en realidad no se 'angosta' como nos muestra la perspectiva, o que un poste de alumbrado a mitad de cuadra, aunque parece lejano y no lo he medido, es idéntico al que tengo a mi lado. Esta certeza 
permite hacer inferencias del tamaño relativo de las diversas cosas que me enfrentan y me permiten 'construir la escala' del lugar o del paisaje. La formación perceptiva de constancias requiere no sólo 'aprehender' las regularidades del entorno sino aprender a leerlas $y$, en su defecto, aprender a disponer de claves que permitan establecer las relaciones entre las cosas y que ellas establecen con nosotros. La 'constancia' se afinca como parte de la experiencia inconsciente e instantánea del proceso de percepción espacial. En éste, los sistemas analizadores (identificación, organización, memoria, asociación, etc.) que nos facultan para advertir que algo es distinto de su fondo; para interpretar los datos sensoriales del medio; y para atribuir valor y significado ( unicidad de un conjunto o el rol de algo en el todo), nos aseguran su localización en varios planos comprensivos (en profundidad y estructuración con lo demás) y , con ello, nos entregan la 'escala' en referencias dimensionales recíprocas o respecto del observador.

Entonces la 'constancia' - y su derivada, la escala - apoyada muy centralmente por el comportamiento del color y de las texturas en la atmósfera (que le da gradiente) y por las sombras, es la que nos permite ver 'en profundidad' - aún a quienes tienen visión monocular - ya que las distancias y las dimensiones de las cosas sería un 'constructo' que no corresponde exactamente a la imagen retiniana ni pertenece a las cosas mismas (므).

Pero, para que opere eficazmente la constancia que postulamos como fundamental, se requiere adicionalmente la presencia de 4 relaciones topológicas ( $\underline{6})$ : suficiente proximidad; suficiente separación; cierta sucesión (o regularidad); y clausura (real / sugerida, 0 indicación de dominio). Ellas hacen que reconozcamos algo como perteneciente a una estructura o a un sistema, o bien como ajeno a él. (Las cosas aisladas, por su parte, en sí mismas no contribuyen al sentido de escala y profundidad, sino en alguna alusión a un contexto. Se rigen por sus propias leyes - como la ley de Rici que postula que se intensifican atributos por la sola pequeñez o aislamiento; o de la Gestalt (ㄱ) que apuestan al poder inductor de la configuración-, las que habrán de considerarse para la lectura y decodificación de los elementos particulares).

\subsubsection{La Orientación perceptual y los 'Mapas Mentales'}

Se sostiene que, para orientarse, el individuo opera las señaladas relaciones topológicas necesarias a la 'constancia', mediante las que es capaz de establecer centros (o focos), direcciones ( $y / 0$ ejes, pautas) y áreas (o campos de comprensibilidad e influencia, o de referencia perceptiva) ( $\underline{8})$, conceptos con los que organizamos el conjunto de presencias. Esta especie de subestructura virtual perceptiva que construimos sobreponiendo una noción geométrica simple, funciona como hipóstasis y nos permite componer los fragmentos en unidades coherentes y orientarnos. Por lo dicho, la ausencia de constancias impediría el fácil establecimiento de estas estructuras. Y, no obstante ser una especie de esquema plano (a 'vuelo de pájaro') y abstracto, se opera perfectamente a nivel de observador concreto.

Pero, una vez recorridos y experimentados, los esquemas comprensivos devienen en 'mapas' significativos, 'mapas' personales que no son homogéneamente neutros como 
los anteriores ni coincidentes con la realidad y que nos hacen ver determinado espacio como un lugar. Los 'mapas' mentales son expresión de vivencias y se acomodan a la predominancia de unos rasgos por sobre otros en la experiencia, conforme van teniendo significado para el observador como referentes. La capacidad de los elementos de un estímulo de reflejar mejor las condiciones del ambiente (iluminación, sombras, por ejemplo) y de manifestar con mayor elocuencia las direcciones en que sus formas se desarrollan, hace que sus rasgos mantengan prevalencia en la percepción y en el 'mapa'. De aquí que, a escala urbana, las superficies como límites del espacio - o las sucesiones tendientes a ser percibidas como tales - permiten buena orientación ( $\underline{9})$.

En el ámbito de la sicología ambiental, los mapas mentales están referidos como 'mapeo cognitivo'. Es un modo de adquisición, almacenamiento, decodificación y utilización de la información, para la comprensión y uso del ambiente. Representación simbólica que surge de la experiencia visual, simplificada en un esbozo mental ( $\underline{10})$. Dependiendo del modo de movilización en que nos desplacemos por la ciudad, el mapa que formemos será diferente. Hemos demostrado en sendos estudios perceptuales con pasajeros de autobús y de Metro (11), que las percepciones y mapas finales son totalmente diferentes entre sí y respecto de los que construyen los peatones habituales en los mismos sectores urbanos. De aquí que la orientación que se alcance en una u otra situación sea diferente. A su vez, las estructuras del entorno tienen un doble papel en la orientación y en la formación de mapas mentales. Por una parte, la percepción aún con clave mínimas, descubre la noción que está en la base de la estructura en que nos encontramos (por ejemplo, un redondel, o un damero) o bien 'sobrepone' una conocida para adoptar confianza; y por otra, una vez captada dicha estructura, ésta induce la lectura y las expectativas sobre el resto del lugar.

De modo que el complejo material que llamamos ciudad es un desafío, especialmente a la percepción visual. Induce comportamientos en la medida que puede ser entendida y siempre que seamos capaces de orientarnos y de descifrar sus significados.

\section{2.- Presencia del factor Percepción Visual en las publicaciones sobre Movilidad Urbana Sostenible}

\section{1.- Los postulados explícitos}

Analizado primero en forma general las publicaciones disponibles, se advierte que, comprensiblemente por su carácter de 'cruzada', se da prioridad a la necesidad de hacer consciencia sobre la congestión y la contaminación -y todos sus problemas asociados - que producen las crecientes cantidades de vehículos particulares, mayoritariamente utilizados individualmente e indiferentes al seguro colapso. $Y$ en consecuencia también, que el enfoque se dirija certeramente a intentar disminuirlos con medidas específicas y de doble efecto, focalizadas en ambos problemas: por una parte, se intenta producir descongestión promoviendo el transporte público, lo que contribuye de paso a descontaminar; y - paralelamente- descontaminar, alentando el desplazamiento peatonal y ciclístico, lo que evidentemente descongestiona las vías. 
Llama la atención sin embargo que, en cuanto a la congestión de las vías - y tomando en consideración la idiosincrasia del urbanita contemporáneo, mentalizado hacia el menor esfuerzo y optimización del tiempo -, no se analicen otras formas de movilidad motorizada como son las motocicletas, o la posibilidad de presionar sobre la industria en la producción de automóviles uni o bipersonales, vehículos que reducirían a la mitad el espacio de carril que ocupan los automóviles actualmente. $Y$ en cuanto a la contaminación, haber revisado la posibilidad de vehículos eléctricos o solares, también de menor envergadura.

La precisión y exhaustividad con que se abraza - en diversos niveles de criterios de planificación y de gestión- el postulado orientado a la combinación de transporte público con desplazamiento no motorizado, parece sin embargo no haber dejado aún el tiempo necesario para abordar con profundidad otros aspectos - como la PV que nos ocupa - los que no cabe duda serán centrales al éxito de las medidas.

No obstante, de alguna manera se alude a la percepción globalmente y a la PV aunque indirectamente y sin entrar en detalle - en algunas secciones de los textos fundamentales:

1.-Cuando se defiende la conveniencia de tender hacia una ciudad más compacta, dado el hecho de que la ciudad dispersa o difusa estaría generando costos perceptuales y pérdida de plusvalía ;

2.-O cuando, refiriéndose a la evolución de la mancha urbana, se consigna la acuñación en Norteamérica del término "environmental deprivation" referido a que la falta de variedad en el ambiente tendría un efecto psicológico en los niños (12).

3.-También, cuando se invita a superar el 'zonning', por su efecto directo en la homogeneidad y monotonía del ambiente, dejando zonas segregadas y faltas de vida cotidiana, atentando contra la 'biodiversidad urbana' (13).

4.-O cuando se dice que tanto las ciclovías como las vías peatonales que se aconsejan para la ciudad 'paseable', deben ser 'seguras y atractivas' $(\underline{14})$;

5.-Asimismo, entre el conjunto de medidas sugeridas para conseguir el objetivo, cuando se incluye -entre otras- volver a la fachada continua, al comprobarse que las discontinuidades y sitios eriazos, baldíos o inanimados "disuaden al peatón".

\section{2.- Otros aspectos que pueden tener efecto visual específico}

El material bibliográfico da muestras entonces de alguna preocupación por el factor perceptual, en general, pero más escasamente por su dimensión visual, aunque algunos de los aportes, criterios y sugerencias, invitan a interpretar el efecto perceptivo que tendrían. Esta falta de enfoque desde otras disciplinas humanísticas se delata cuando se echa de menos un " mayor diálogo multidisciplinal": " No hay por ejemplo, ninguna aproximación sociológica o antropológica, perspectivas también claves para explicar la suburbanización"(吕). 


\section{RUVSTADE \\ URBANISMO}

http://revistaurbanismo.uchile.cl

\subsection{1.- Visualidad y efecto actitudinal}

Más allá de alusiones indirectas en las que incidiría la percepción global ("la ciudad que conocemos causa un insostenible stress" ; "el concepto de ciudad compacta contradice el profundo aprecio por la vida suburbana y semirural en el Reino Unido" ( $\underline{16})$ ), hay otras que, aún en el mismo tenor de generalidad, plantean sin embargo una tesis respecto a que la cualidad visiva del ambiente pudiera favorecer previsibles actitudes:

(Entre los principios para un desarrollo sustentable, se incluye:)

"Principio 2= Necesidades humanas.

"El punto de partida para un desarrollo sostenible es la satisfacción de las necesidades básicas, (entre otras enumera) un entorno placentero. Las metas sociales y medioambientales, usualmente se refuerzan mutuamente."

" Un entorno peatonal atractivo, seguro y bien previsto, trabaja a favor de la estabilidad social y de la creación del sentido de comunidad"

"Principio 3= Estructura: sobre la base de redes eficientes de energía y movimiento, permeabilidad peatonal y acceso.

(Refiriéndose a la tensión entre 'cul-de-sac' y pasajes peatonales en red).

"La respuesta está en un buen esquema, asegurando que los propios residentes hacen la vigilancia y evitan lugares peligrosos". " la cantidad de personas y el trazado son factores que contribuyen a su uso" $(\underline{17})$

Otros criterios de sostenibilidad, también referidos a aspectos generales que lindan con la PV, se refieren a actitudes que, más precisamente, promueven comportamiento social cooperativo, a partir de atributos visivos que, en último término, provocarían condiciones de deseabilidad y de empatía y, de allí, la estabilidad del modelo. 
1.- (Criterios de sostenibilidad -aceptados por diversos autores- ordenados según jerarquía de necesidades de Maslow. Después de 1: Provisión de las necesidades físicas, añade:)(18)

2.-Seguridad y protección

Un entorno visual y funcionalmente ordenado y controlado.

3.- Conductividad social.

Un entorno socialmente conductivo

Sentido de comunidad y pertenencia a un lugar

4.-Buena imagen, reputación y prestigio.

Un lugar que provoque la sensación de confianza y de fortaleza.

Que de estatus y dignidad.

5. - Oportunidad de ser creativos.

Lugar que brinde oportunidad de dar forma y modelar lo propio, individualmente. Que de oportunidad a las comunidades de modelar participativamente su distrito.

En ellos se dice qué, pero no está claro el cómo desde el fenómeno de la visualidad. Esta tendencia a asumir efectos actitudinales a la condición del diseño con fuerte componente visivo, aunque sin especificar, se ve también reflejada cuando se analizan algunas soluciones suburbanas realizadas bajo los paradigmas del neo-urbanismo:

(Refiriéndose a los patrones de vías internas, al comparar Kentlands y Laguna West, en los EE.UU, se dice: ) "Los 'pasajes de servicio ( alleys) proveen acceso visual por detrás a las viviendas, . A pesar de que 'probablemente' reducen la privacidad, promueven a una vecindad más casual y espontánea".(19)

\subsection{2.- La variedad visual como valor}

Del mismo autor, encontramos otras observaciones donde sí puede deducirse estando implícito - un factor visual que, claramente, provoca determinado efecto. En el siguiente caso se puede advertir el valor de la variedad visual para capturar la atención, despertar el interés y provocar acciones de lectura más atenta, que crean el vínculo primero que es necesario a la empatía:

(Al analizar los suburbios de Elmswood, Kentlans y Laguna West) (20)

Compara el 'sentido' de casas hechas todas de una sola vez, con y sin un estilo forzado, respecto de aquellas construidas con la diversidad que da el tiempo. "El crecimiento incremental da una sensación más hogareña, allí donde las calles tienen más pequeños jardines y se sienten más angostas".

En la defensa del modelo de la sostenibilidad, varios autores echan de menos la variedad por otras múltiples razones - no siempre de efecto visual-, entre ellas la

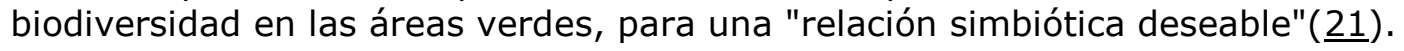




\subsection{3.- La visualidad e Imaginabilidad: necesidad de 'noción' organizativa}

La presencia o ausencia de una 'noción organizativa' que de las pautas para estructurar los elementos del entorno, tiene enorme importancia en el proceso de la comprensión, orientación e imaginación posterior de un lugar.

Entre los criterios de sustentabilidad que se enuncian como comúnmente aceptados y enunciados a partir de Maslow - ya citados en parte-, los que se presentan acompañados de las cualidades ideales de la ciudad, se agrega un último el cual sería el único que tiene directa incidencia en la percepción visual, y que implica la necesidad de una 'noción organizadora'.

\section{Un entorno estéticamente placentero. \\ Un entorno bien diseñado \\ Un lugar fácilmente 'imaginable' en lo físico.}

Y agrega el autor algo que resulta esencial para lo que venimos postulando, al asociar a la cualidad de poseer una estructura basada en una noción clara, el siguiente criterio comúnmente aceptado de sostenibilidad : " ( la ciudad) requiere una Cualidad VisualFormal;... Imaginabilidad de la ciudad como entidad total y de sus parte, barrios y distritos.... Provisión de sentido de centro y de lugar"...... "una estructura que genere una alta legibilidad e imaginabilidad de la forma del asentamiento".

En el mismo sentido, se valora el orden y la existencia de una clara 'noción organizativa' con cualidades visualmente explícitas, para conseguir los efectos de eficiencia funcional que se esperan, como en el caso específico siguiente:

"Las rutas principales deben irradiar desde centros o paradas con una cualidad de diseño centrada en la mínima deflexión respecto de la ruta en línea recta, (pero) con variedad y atractivo para la potencial experiencia de caminata y evitando puntos de intimidación." $(\underline{22})$

Más allá del sentido de orientación, de comprensibilidad y de facilitar la operación de la ciudad mediante la formación de los 'mapas mentales'; es decir, más allá del valor visivo y de cualidades de la presencia de 'noción' que habíamos anticipado, nos encontramos que también a esta pauta estructural, se le atribuye otras cualidades, que pueden deberse a la misma comprensión facilitada:

"Un estudio midió el 'sentido de comunidad' en suburbios en sprawl y en desarrollos planificados cercanos; los claros límites de estos últimos, sus espacios públicos y sus centros con claras conexiones peatonales, favorecieron mayor satisfacción y sentidos de comunidad. (Glynn 1981). Otro comparó la satisfacción de los usuarios de áreas de mono uso con áreas mixtas, donde estos tenían un mayor sentido de comunidad". 
http://revistaurbanismo.uchile.cl

(Nasar and Julian 1995).(르)

No obstante estas constataciones, el valor social de la visualidad y su utilidad práctica han sido poco discutidos a niveles técnicos en el ámbito del urbanismo.

\subsubsection{Visiones sobre la ausencia de 'noción organizativa' y sus efectos}

Varios autores lamentan la pérdida de una estructura que permita detectar una clara noción organizativa en las periferias con calidad suburbana. Refiriéndose al 'sprawl, recuerdan que el término viene de una actitud del cuerpo, lo que no es necesariamente peyorativo, aunque hoy se asigna para cualquier cinta de borde y para cualquier retazo de baja densidad, cuyo único elemento en común es la "carencia de forma"(24).

Ewing afirma que Gordon y Richardson caracterizan fuertemente lo compacto como "monocéntrico", asignando el concepto de sprawl a las más diversas realidades. No obstante, asegura que éste no es malo en sí mismo, sino por el 'desperdicio' (de espacios) y por el hecho de que no tiene imaginabilidad, cuando sostiene:

" No está claro en qué número de centros el policentrismo desaparece y el sprawl comienza (Gordon \& Wong 1985). Igualmente elusiva es la línea entre un desarrollo a 'salto de rana' y uno desarrollo discontinuo económicamente eficiente; o entre una

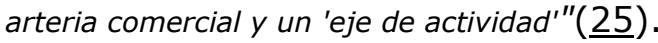

Pero esa característica general de las zonas dispersa, cual es la de carecer de una noción estructurante en sí misma y también en relación aquella de la ciudad de la que forma parte, para otros autores parece cobrar un valor propio, descubriendo en ello varios signos positivos, (haciendo nosotros la salvedad de que no se oponen, ya que no consideran el beneficio que significa un ambiente que refuerce nociones, por las razones que hemos comentado).

G. Dematteis reconoce, por ejemplo, que existirían diferencias nocionales entre la ciudad compacta y las suburbanizaciones dispersas, que son derivadas de la cultura y desarrollo, o también del crecimiento de nodos especializados con o sin dependencia funcional de los centros tradicionales. Estima sin embargo que ello no sería negativo, ya que las relaciones hoy no son espacio-visuales, puramente, sino también de otro tipo. Atribuyéndolo al gran cambio producido en la década del '60," marcado por el tránsito entre la sociedad fordista y posfordista,... "caracterizado por la relajación de las relaciones jerárquicas, por la flexibilidad de la organización productiva y del trabajo, por la multiplicación de las relaciones horizontales y por la aparición consiguiente de las indentidades o especificidades locales", y propone:

"A fin de hacer representable esta nueva dinámica y las formas espaciales que se derivan de la misma es preciso sustituir la idea clásica de posición geográfica relativa o 


\section{REVSTADE URBAANISMO}

http://revistaurbanismo.uchile.c

absoluta -que se refiere a un espacio homogéneo y continuo - por una posición relacional que hace referencia a otro tipo de espacio virtual discontinuo y heterogéneo. Se trata de un espacio que varía de un lugar a otro según la disposición y superposición de las diversas redes de relaciones económicas, culturales y políticas que lo atraviesan. Esto significa que todo lugar y todo sujeto localizado, puede pertenecer contemporáneamente a redes diferentes que interactúan a escalas distintas". (21)

Postula así una relectura y valoración de la suburbanización y de la periurbanización, ya que hasta ahora a las periferias se le achacan todos los males imaginables, como 'espacios atópicos' (sin lugares). En cambio hoy la periferia se elige para habitar por cualidades que el centro no tiene. Dice:

"todo esto nos hace reflexionar sobre el hecho de que hasta hace poco tiempo, al menos en Europa, la imagen negativa de la periferia urbana ha sido producida por una cultura hegemónica cuyos representantes se identificaban con el 'centro' donde solían habitar." (Etapa fordista, estratificada socialmente. La mezcla actual destruye la metonimia centro-periferia.). (27)

No obstante esta ausencia de estructura, reconoce cualidades formales de las periferias que ya no se refieren a la ciudad madre sino que son otra cosa. "A partir de los '80, la periferia es (simplemente) ciudad sin centro". Para él la ciudad compacta del fordismo, se identificaba por edificaciones monótonas sin orden ni forma, siendo éstos por ello 'atópicos' para él; barrios del 'conflicto constructivo', al convivir allí toda expresión. En tanto la periferia del posfordismo: presenta el atractivo de mosaicos de lugares, con cualidades formales propias; lugares que permiten la afirmación individual.

"...siguiendo esta tónica, se llega a invertir completamente la imagen negativa de la periferia, afirmando que durante los últimos años, ella ha sido 'metrópolis', en el sentido etimológico de 'ciudad madre', generadora de nuevos modelos culturales, sociales y políticos."

Esta postura disidente es de la mayor importancia. Nos preguntamos cómo se están formando hoy los 'mapas mentales' en la generación postracionalista.

\subsubsection{La variable energética y su efecto visual}

La dispersión de la ciudad - discutida más arriba-, que es el centro del debate para el modelo de sostenibilidad que se apoya en una movilidad alternativa al vehículo particular individual para evitarla, tendría sin embargo, el atributo positivo de facilitar la incorporación masiva de la energía solar doméstica y otras formas de energías 
http://revistaurbanismo.uchile.c

alternativas, que también contribuyen a la descontaminación (28). Ello tiene efecto visual.

Dichas energías, por requerir condiciones físicas precisas - como predominio de la masa o soterramiento; colores oscuros, tendientes al negro, en convivencia con superficies vidriadas; grandes planos inclinados y volúmenes de acopio, cuando no grandes estanques estratificadores, elementos captadores o molinos, aún si las celdillas solares hacen su costo accesible - todo lo cual tendrán un impacto visual 'artefáctico' que de por si imprimirá carácter, distinto al tradicional.

Estas construcciones tienden a desarrollarse mayormente en extensión y requieren de espacio en derredor si se opta por el reemplazo integral de energías, lo que implica, además de invernaderos, digestores para producir gas metano, incentivando la tendencia a una ciudad dispersa. Lo anterior se consigna en el últimos de los 'principios' que, para la sostenibilidad urbana, propone Burton.

"Principio 6: Una estrategia para la energía: maximización de ganancia solar y ahorro

" Favorecer divisiones prediales hacia el sol con un ángulo máximo de 450; evitar los solares sombríos.

" Evitar los procesos (constructivos) que incorporan energía o pérdida de energía, reutilizando y haciendo construcciones durables, con recursos locales, de baja altura, y que aprovechen las redes.

Esta postura filosófico práctica, modelará finalmente con seguridad un determinado 'carácter' urbano en extensión, de claro impacto visual.

\subsection{6.- Conflictos visuales entre ciudad y vías de transporte}

La naturaleza y envergadura de las vías urbanas y carreteras hoy, así como su aparición en el tiempo respecto de lo edificado, crean muchas veces situaciones de compatibilidad que en el pasado no existieron. Si antiguamente las masas construidas determinaban con su mismo volumen los vacíos que eran cauce de los flujos, en la actualidad, y especialmente en la periferia, muchas veces aparecen antes las vías y carreteras - prolongaciones o nuevas conexiones desde la ciudad - las cuales imponen invariable su propia lógica y geometría eficiente, generando una lotificación que queda disponible con las más variadas tramas y angularidades (29).

Aunque sin estricta preocupación por este caos visual que ocasionan sino más bien señalando un conflicto funcional, hay señales en los textos de que existe preocupación al respecto, cuando refiriéndose a las ciudades progresivamente rodeadas por una urbanización dispersa, se dice: "es en los ámbitos suburbanos donde los problemas de coordinación aparecen con más claridad"( $\underline{30})$. Y se añade: 
http://revistaurbanismo.uchile.cl

"Y ese conjunto se basa precisamente en el desarrollo y articulación de un sistema comarcal y regional de carreteras y autopistas que permiten la conexión con el centro urbano principal..."

" las carreteras deben cumplir, a menudo, un doble papel. Por una parte son el elemento de conexión de todo el sistema de asentamientos, la red de comunicación que permite el funcionamiento del conjunto. Por otra, constituyen a menudo el eje estructurante del incipiente desarrollo urbano de muchas áreas, y cumplen, por tanto un papel cualificador del espacio urbano".

Reconociendo en ello una "doble tensión" - distinta, sin embargo, a la tensión visual necesaria para provocar fuerzas neguentrópicas' e interés visual - propone una metodología para abordar estos conflictos.

\subsubsection{Soluciones de articulación vial y su implicancia visual}

Algunas soluciones de diseño que se proponen para el punto de la articulación de flujos, de funciones o de elementos de distinta naturaleza, sugieren una implicancia visual, lo cual, en alguna medida es reconocido, como en los siguientes casos que se refieren al diseño de diversos tipos de intersecciones, primero 'a nivel' y luego a diferente nivel:

\footnotetext{
"Las intersecciones giratorias o glorietas (cualidades).... y permiten interesantes tratamiento 'paisajísticos' - Se consideran muy recomendables para marcar el comienzo de una travesía, aunque son incómodas para los peatones."

"...Los pasos a distinto nivel son caros, incómodos para los peatones, de fuerte impacto visual y, a menudo, constituyen una barrera psicológica para los peatones .

"... En lo referente a las bandas longitudinales de peatones, aceras y arcenes, parece importante desarrollar criterios de dimensionamiento, diseño (elevadas o no, separadas de la calzada por un arcén, vallado, etc.,) materiales, plantaciones, etc." (31)
}

Al respecto habría que señalar que, respecto a las glorietas, la apreciación negativa que se les atribuye por parte de los peatones, no es siempre tal, cuando éstas son suficientemente amplias - como deben serlo para ser eficientes- ya que en ese caso cruzar la calzada no es diferente a cruzar cualquier otra; y, llegados a ella, pueden ser atravesadas en secante y sin tener que rodear, si se las diseña convenientemente. Perceptivamente ellas son positivas y orientadoras, más allá de que pueden albergar múltiples motivos urbanos simbólicos o funcionales.

En cuanto a los pasos peatonales sobrenivel, es evidente que no ha habido buenos diseños. Pero si se suaviza su pendiente con rampas que no sea desalentadoras y se les integra mejor en su desplante desde el flujo peatonal, deberían progresivamente ir teniendo aceptación y mayor uso. Las protecciones a las bandas peatonales, por su parte, sí que son desalentadoras y llevan a 'odiar' la ciudad mientras ofrezcan un tratamiento como para rebaño de animales, que hace rebelarse el espíritu libertario del 
http://revistaurbanismo.vchile.c

transeúnte. Cualquier límite ya sea de guía o de protección, debe contemplar este aspecto, privilegiando aquellos diseños menos invasivos o intrusivos. Lo mismo sucede con las proposiciones de acondicionamiento frente al ruido que se ofrecen aquí para las carreteras: se enumeran sin mayor consideración al efecto de pertenencia, pertinencia, goce visual, predisposición positiva al ambiente, integración, etc.:

"la influencia del perfil longitudinal y de la sección transversal de la carretera en la producción y propagación del ruido. ( pendiente, ventajas en trinchera, etc).

" la eficacia de diferentes elementos para interceptar la propagación del ruido ( como diques, pantallas sónicas, vegetales, etc.).

$(\underline{32})$

Finalmente se abordan los acondicionamientos ópticos, señalando algo que para la PV resulta central:

\begin{abstract}
"Esta técnica se basa en el hecho empíricamente contrastado de que el ambiente, independiente de la geometría de la vía, tiene una gran influencia sobre el comportamiento de los conductores; y de que la presencia o visibilidad de potenciales conflictos induce a una reducción de velocidad".

" Según estos análisis, el automovilista adapta su conducta al ambiente que percibe... debiendo hacerse explícito, evidente...No se trata de crear decorados artificiales,... sino de hacer legible el ambiente, visibles al automovilista".
\end{abstract}

La precedente observación valida la fuerza de la percepción. Pero resulta evidente que, además de constatar el hecho empírico, se haría necesario describir los atributos visuales que resultan más eficientes.

\title{
3.- Efectos Perceptuales que incidirían en el éxito de algunas estrategias para una Movilidad Sostenible
}

Algunas estrategias presentes en la bibliografía, sugieren detenerse a considerar con mayor profundidad aspectos perceptuales -holísticos o visuales- para su mayor eficacia, proyectando el efecto comportamental que resultaría de su aplicación, ya que puede llegar a la larga, a influir en contrario. Los abordaremos en el orden aleatorio con habida consideración a la importancia que le asignan los autores.

\subsection{Características del diseño de las Vías como promotores y disuasores del modelo}

Para detractar a la ciudad dispersa y la extensión que están adoptando las manchas urbanas, se esgrime el hecho de que las vías y autopistas y toda la infraestructura viaria, adquiere una enorme extensión. Más allá de las razones que se dan, con las que no se puede más que concordar, hay dos hechos perceptuales que aconsejan evitar su 
http://revistaurbanismo.uchile.cl

mayor extensión. Pero no sólo la extensión sino también el ancho que adquieren obligarán a preocuparse de otros factores, especialmente a quienes postulan por una ciudad compacta en lo que dice relación con las vías de transporte colectivo. Los efectos a que nos referimos son: el comportamiento basado en la expectativa de origen visual; y el comportamiento basado en la necesidad de individuación en todo ser humano, el cual muchas veces se origina como reacción al paisaje visual. Frente a cada uno, observamos:

\begin{tabular}{|l|l|}
\hline Vías muy largas en la ciudad dispersa & $\begin{array}{l}\text { Expectativa: } \\
\text { La percepción inalcanzable de lo que 'falta por } \\
\text { recorrer lleva por sí mismo a acelerar } \\
\text { Individuación: } \\
\text { En la democrática autopista, la única distinción es la } \\
\text { 'cilindrada', que se demuestra acelerando. }\end{array}$ \\
\begin{tabular}{|l} 
Vías muy anchas y con carriles segregados en la \\
ciudad compacta. (por la jerarquía de vías y por el \\
privilegio al transporte público)
\end{tabular} \\
$\begin{array}{l}\text { Ea vía más ancha hace disminuir la percepción de } \\
\text { velocidad, por lo que, de no haber congestión, se } \\
\text { acelera. } \\
\text { Individuación: } \\
\text { La sensación de 'rebaño', por los demarcados carriles } \\
\text { paralelos y exceso de normas, lleva a revelarse y, si } \\
\text { se puede, a infringirlos. }\end{array}$ \\
\hline
\end{tabular}

\section{2.- Ciudad Compacta / Ciudad Dispersa....o hasta 'Difusa'}

Las consideraciones que comparativamente se exponen en los textos inclinan la balanza - desde el punto de vista técnico - hacia una ciudad compacta aunque de un modo ecuánime, se da cuenta del debate suscitado. Desde el punto de vista puro de la percepción general y visual, la balanza se inclina en igual sentido.

La ciudad compacta - planificada o formado existencialmente en el tiempo - tiene la posibilidad de modelarse con una estructura clara siguiendo patrones y estructuras. La experiencia en éstas, ya sea kinésica o visual, a su vez contribuye a la formación o reforzamiento de nociones estructurales espaciales. Éstas, si bien se forman en la experiencia perceptual, informan de un modo iterativo las percepciones secuentes y son la base sobre las que se arman los Mapas Mentales mencionados. La ciudad dispersa por su parte, si está formada de núcleos compactos con patrones coherentes, éstos tienen la posibilidad también de apoyar la formación de nociones y de imprimir un soporte para el mapa mental dependiendo de la coherencia de lo núcleos con el todo, no obstante que la composición del total puede permanecer confusa y descifrada por retazos.

Desde este enfoque, la llamada ciudad difusa - la de salpicaduras o manchas - que crece sin acuerdo social, y que muta tan radicalmente en lapsos pequeños de tiempo, es incapaz de apoyar la formación de nociones formales y espaciales y, por lo tanto, de apoyar la formación de Mapas eficientes. Si bien los aborígenes de América se orientaban en el bosque y en lo agreste que adolece de gran variedad, formaron sus nociones porque precisamente la naturaleza tiene al mismo tiempo la mayor 
regularidad, aquella de que carecen los nuevos habitantes de la suburbia. La periferia, a la falta de unidad, carácter e identidad, suma la falta de cohesión - lógica, funcional, pertinente - de sus partes.

Esta confusión por falta de 'constancias' en la ciudad difusa obedece a una lógica vital que resulta en espacios con otro 'ADN', por decirlo de algún modo. A diferencia de las ciudades compactas de lenta formación, en que el cauce o vía surge simultáneamente y es efecto de la masa construida, en la totalidad de las zonas de expansión urbana es la vía -planificada o no - la primera en existir con su propia lógica y configuración, a la que se adaptan las construcciones y subdivisión predial. Ello redunda en variadas y caprichosas tipologías prediales que contribuyen a la baja comprensibilidad, variedad que se agudiza por el hecho de que la menor densidad y amplitud impone destacarse con la una búsqueda de individuación, sumando a lo anterior la mayor diversidad edilicia. Es en la ciudad difusa donde más difícilmente se encontrarán suficientes 'constancias' que promuevan la comprensión del entorno.

En consecuencia, desde el punto de vista de la visualidad, a las inconveniencias que presenta la ciudad difusa y la dispersa referidas en los textos a la movilidad sostenible, se suman efectos que dificultarían la comprensión y orientación en ese espacio urbano. Si se trata de hacer la ciudad 'vivible' y de reducir al mínimo la circulación vehicular privada, la desorientación y la ausencia de mapa mental que ocasiona el modelo, provocará por el contrario recorridos ineficientes o de tanteo, dificultando presuponer atajos e incrementando su ajenidad.

\subsection{Las Propuestas para la Gestión de la Demanda de Movilidad: reflexión visual y sus efectos}

Pueden también analizarse desde la percepción y la visualidad, el conjunto de medidas estratégicas que se estiman con alto efecto en la disminución de la movilidad motorizada en vehículo privado ( $\underline{33})$. Dado que en los textos se exponen detallada y suficientemente descritas, aquí se enumeran por conveniencia en una redacción abreviada y se comentan, a veces, integradas entre dos o más de ellas. (Las observaciones que se acompañan suponen un comportamiento general, aunque, como hemos dicho, cada caso dependerá de su situación y filtro cultural).

\begin{tabular}{|c|c|}
\hline Medida estratégica & Factor perceptual: \\
\hline $\begin{array}{l}\text { a) } \\
\text { " Evitar salir" / } \\
\text { "Proveer la mayor cantidad de servicios co } \\
\text { alcance peatonal o ciclístico". }\end{array}$ & $\begin{array}{l}\text { Recluir al ciudadano a su barrio, es discutible porque, además } \\
\text { de difícil, inhibe la capacidad de exploración; disminuye la } \\
\text { seguridad de movimientos; circunscribe la formación de } \\
\text { nociones espaciales, de claves de orientación y de mapas } \\
\text { mentales; limita la reacción creativa frente a imprevistos, } \\
\text { rasgos todos necesarios para el individuo contemporáneo. }\end{array}$ \\
\hline $\begin{array}{l}\text { b) } \\
\text { "Incentivar el uso del Transporte Masivo". } \\
\text { " Para su fluidez y rentabilidad social, éste } \\
\text { debe ser: } \\
\text { lineal -directo, } \\
\text { en carril privilegiado, }\end{array}$ & $\begin{array}{l}\text { Transporte de superficie: } \\
\text { Desde un viaje frecuente en autobús, la percepción de la } \\
\text { ciudad se fija de dos maneras ( } \underline{34}) \text { : por caracteres } \\
\text { homogéneos que la velocidad nivela hasta acentuar ese } \\
\text { carácter; y por la interrupción que produce la significancia de } \\
\text { hitos visuales, los cuales, de estar muy cercanos, se anulan } \\
\text { como tales o se perciben en conjunto. La escala y }\end{array}$ \\
\hline
\end{tabular}


http://revistaurbanismo.uchile.cl

\begin{tabular}{|c|c|}
\hline $\begin{array}{l}\text { rítmico - con paradas 'animadas' } \\
\text { cada 500, } 600 \mathrm{~m} \text {. } \\
\text { " flanqueado de zona densa. } \\
\text { "Superar el 'zonning'. }\end{array}$ & $\begin{array}{l}\text { singularidad de dichos hitos es central para facilitar la } \\
\text { orientación y localización. } \\
\text { Su linealidad hace que muchas ciudades concentren varias } \\
\text { tipologías de tráfico en el mismo cauce viario privilegiando la } \\
\text { fluidez del transporte masivo, al que se asigna generalmente } \\
\text { al carril central para no ser interrumpido. Esta modalidad deja } \\
\text { al TP en medio de un mar de carriles saturados, donde la } \\
\text { velocidad aumenta la nivelación entrópica de la monotonía } \\
\text { que lo separa del paisaje que podía ser significante y } \\
\text { estimulante para asumir el modelo. } \\
\text { En estos trayectos largos y acompañados de monótonas } \\
\text { bandas motorizadas, el denigrado 'zonning' puede resultar } \\
\text { una ayuda a la ubicación espacial. (Estas anchas bandas } \\
\text { motorizadas son un límite perceptual que se trata más } \\
\text { adelante). } \\
\text { Transporte subterráneo: } \\
\text { Desde la habitualidad del Metro se ha comprobado * que se } \\
\text { pierde dramáticamente la orientación y se adquiere una } \\
\text { imagen fragmentada y desescalada que inhibe un mapa } \\
\text { mental útil. Las zonas intermedias, aún conocidas, van } \\
\text { perdiendo significado e imagen, reemplazado por los nombres } \\
\text { de estaciones, mientras los trayectos se internalizan como } \\
\text { puro tiempo rítmico. La percepción de las ciudades } \\
\text { homogéneas es menos afectada que la de ciudad en } \\
\text { 'zonning', donde se emerge en áreas de carácter muy } \\
\text { diverso. } \\
\text { Esta 'emergencia' a la superficie, por sí misma plantea } \\
\text { problemas perceptuales. Los estudios exploratorios muestran } \\
\text { que se orientan mejor quienes emergen en espacios abiertos } \\
\text {-como parques - frente a quienes lo hacen en espacios más } \\
\text { estrechos o interiores. }\end{array}$ \\
\hline $\begin{array}{l}\text { d) } \\
\text { "Promover áreas de uso mixto". } \\
\text { " mayor autosuficiencia con variada } \\
\text { tipología edificatoria y de tamaños". }\end{array}$ & $\begin{array}{l}\text { Perceptualmente, las áreas mixtas si son además } \\
\text { tipológicamente variadas tienen efectos opuestos: Por una } \\
\text { parte, si guardan suficiente o algún grado de identidad, o } \\
\text { bien una estructura que le de unidad nocional (para ser } \\
\text { comprendida como totalidad), la variedad propia de estas } \\
\text { zonas se hace claramente positiva contribuyendo a la lectura } \\
\text { específica de los componentes en un todo no amenazante, } \\
\text { por basto que sea. } \\
\text { Si por el contrario, la diversidad en todo sentido se impone } \\
\text { sin brindar claves de ningún tipo para la asociatividad y para } \\
\text { la lectura de sub-zonas o del total, el efecto perceptual } \\
\text { general se hará 'entrópico' *, funcionando negativamente, el } \\
\text { mecanismo de nivelación: a pesar de la variedad, se percibirá } \\
\text { parejamente caótico. }\end{array}$ \\
\hline & $\begin{array}{l}\text { La lectura del 'carácter' de una zona, entre otros, es } \\
\text { importante para la orientación, como también lo es reconocer } \\
\text { 'tensiones' del lugar, o sea, esas fuerzas de vinculación o } \\
\text { pertenencia entre elementos, de correspondencia, etc., de las } \\
\text { que se han identificado } 28^{*} \text {. Atributos como el carácter, } \\
\text { contribuye reforzar actitudes de seguridad y autonomía en el } \\
\text { espacio, mientras las fuerzas tencionales - que son síquicas- } \\
\text { actúan de modo 'neguentrópico'( } \underline{35}) \text {, esto es positivamente } \\
\text { hacia los mecanismos de agudización y de lectura } \\
\text { significativa. } \\
\text { De aquí que la planificación de las áreas de uso mixto }\end{array}$ \\
\hline
\end{tabular}


http://revistaurbanismo.uchile.cl

\begin{tabular}{|c|c|}
\hline & $\begin{array}{l}\text { resultarán perceptualmente positivas, si la variedad buscada } \\
\text { mantiene no obstante un claro carácter y se disponen y } \\
\text { diseñan elementos que mantengan suficiente 'tensión' entre } \\
\text { sí y con el contexto. }\end{array}$ \\
\hline $\begin{array}{l}\text { e) } \\
\text { "Concentración de las densidades } \\
\text { habitacionales hacia los corredores de } \\
\text { Transporte público". }\end{array}$ & $\begin{array}{l}\text { Esta medida por sí misma otorga a los bordes de corredores } \\
\text { un sello distintivo respecto del resto, lo que es claramente } \\
\text { positivo. Sin embargo, estas cintas densas han de acoger } \\
\text { suficiente variedad, controlada hasta el límite de no producir } \\
\text { nivelación y homogenización 'entrópica'. Por lo dicho } \\
\text { anteriormente, las cintas edificadas deben proveer un ritmo } \\
\text { de hitos que posibiliten la localización espacial en el trayecto. }\end{array}$ \\
\hline $\begin{array}{l}\text { f) } \\
\text { " incorporar y privilegiar redes eficientes de } \\
\text { ciclovías" } \\
\text { Éstas deben ser directas, 'atractivas', } \\
\text { seguras. }\end{array}$ & $\begin{array}{l}\text { Las ciclovías, que junto con las vías peatonales, son centrales } \\
\text { al modelo, desde el punto de vista perceptual deben en } \\
\text { primer lugar responder a la propia naturaleza del medio: es } \\
\text { uno de los más ágiles para realizar evoluciones; permite } \\
\text { pasar de un tipo de superficie a otra; se le invierte con } \\
\text { facilidad para tomar la dirección contraria; su liviandad } \\
\text { permite (cargándola) remontar obstáculos o peldaños, etc. } \\
\text { Por ello da la sensación de gran libertad, que otros medios } \\
\text { como el automóvil no proporcionan. } \\
\text { Si bien - como se dice - las ciclovías deben ser 'directas' y sin } \\
\text { obstáculos para su una eficiente movilidad, debieran } \\
\text { idealmente proveer las condiciones para experimentar la } \\
\text { libertad de opciones que la bicicleta permite, a fin de que este } \\
\text { atributo haga preferirla. En todo caso, no debieran ser } \\
\text { excesivamente angostas ni de trazados insistentemente } \\
\text { paralelos. } \\
\text { Se dice también que deben ser 'atractivas'. Una forma de } \\
\text { atraer a los usuarios es la señalada arriba. Otra, es que allí } \\
\text { donde se pueda, su tratamiento y ubicación dé la sensación } \\
\text { de 'paseo' y no de obligación. Su ancho - necesariamente } \\
\text { más reducido que los carriles motorizados- no debe ser } \\
\text { constante e insistentemente paralelo y, en lo posible, ellas } \\
\text { deben ser autónomas, esto es no incorporadas a vías que } \\
\text { contengan otras formas de movilidad. } \\
\text { Esto último tiene relación con el tercer atributo, exigido, es } \\
\text { decir que sean 'seguras' . Obviamente, la percepción de } \\
\text { seguridad se incrementa en la medida de la exclusividad y de } \\
\text { que desde sus áreas adyacentes a lo largo no puedan surgir } \\
\text { sorpresas. } \\
\text { Las ciclovías demarcadas en cauces motorizados o } \\
\text { incorporadas a aceras y señaladas por la calidad del } \\
\text { pavimento, afectan en algún grado la seguridad por la escasa } \\
\text { claridad perceptiva que ofrecen. }\end{array}$ \\
\hline $\begin{array}{l}\text { g) } \\
\text { "Adoptar criterios del 'neo urbanismo', } \\
\text { como la vuelta a redes de tráfico público } \\
\text { claras y jerarquizadas, complementadas por } \\
\text { redes domésticas, arborescentes de accesos } \\
\text { dosificados desde la autopista y no } \\
\text { coincidentes entre si para evitar los cruces } \\
\text { de aquella." }\end{array}$ & $\begin{array}{l}\text { Esta indicación es perceptualmente positiva en cuanto a la } \\
\text { jerarquización y claridad que adquieren las redes principales. } \\
\text { Éstas, como dijéramos, contribuyen a la formación de } \\
\text { nociones reforzando la comprensibilidad del total, al tiempo } \\
\text { que facilita la orientación espacial. } \\
\text { No obstante su complemento - las redes internas menores y } \\
\text { arborescentes - , no contribuyen perceptualmente al mismo } \\
\text { fin, ya que tienden a desorientar, especialmente cuando } \\
\text { integran trazados semicirculares que alcanzan los } 180^{\circ} \text {. } \\
\text { Curvaturas de menor desarrollo no tienen igual efecto. Por } \\
\text { otra parte, las discontinuidades - físicas y perceptuales- que }\end{array}$ \\
\hline
\end{tabular}




\section{REVISTA DE URBANISMO

http://revistaurbanismo.uchile.cl

\begin{tabular}{|c|c|}
\hline & $\begin{array}{l}\text { para ellas el modelo hace inherentes con el fin de } \\
\text { desincentivar el uso motorizado, si bien logran por su trazado } \\
\text { este propósito pueden además aprovecharse para situar allí } \\
\text { elementos o edificios singulares, de modo de que la } \\
\text { prohibición sea enfática al tiempo que 'tensa' y grata. }\end{array}$ \\
\hline $\begin{array}{l}\text { h) } \\
\text { "templado de tráfico" }\end{array}$ & $\begin{array}{l}\text { Hacer una ciudad más 'vivible' mediante recursos que la } \\
\text { pueden hacer invivible, odiosa y hasta peligrosa, no parece } \\
\text { coherente. El 'templado del tráfico' que parece tan necesario } \\
\text { desde los postulados del modelo, debe en lo posible } \\
\text { privilegiar aquellas soluciones que, junto con lograr su } \\
\text { propósito, no restan agrado a vivir en la ciudad. } \\
\text { Medidas como 'angostamientos' imprevistos, discontinuidades } \\
\text { reiteradas, o retornos y quiebres obligados que escapan a la } \\
\text { lógica del trazado, se leen como incoherencia no sólo por } \\
\text { parte de los automovilistas, restando en mucho armonía y } \\
\text { gratificación visual. Otras como los 'lomos' o similares, } \\
\text { inciden menos en la percepción visual, aunque debieran ser } \\
\text { muy evidentes para ser anticipadas. Pero, más importante, } \\
\text { muchas de ellas pueden costar una vida o una casa: en un } \\
\text { incendio o un infarto cardíaco, p. ej., unos minutos hacen la } \\
\text { diferencia. } \\
\text { Otras medidas como las 'glorietas' o las inflexiones del } \\
\text { trazado por curvaturas controladas, templan sin restar fluidez } \\
\text { visual y aportando armonía. O como el angostamiento } \\
\text { homogéneo de todos los carriles, que hace disminuir la } \\
\text { velocidad por su propio efecto perceptual, induciendo a un } \\
\text { control más fino del vehículo, además de permitir destinar } \\
\text { menos superficie a la vía. Todas ellas permiten en caso } \\
\text { necesario, llegar con rapidez apoyando la sensación de } \\
\text { seguridad. Adicionalmente, las 'glorietas' permiten un realizar } \\
\text { eficientes diseños de distribución de distintas direcciones de } \\
\text { tráfico y de modalidades de transporte, haciéndose } \\
\text { suficientemente significativas a la percepción por su densidad } \\
\text { de mensajes, y pueden incorporar jardín o elementos } \\
\text { ornamentales y decorativos, señalando en mejor forma un } \\
\text { foco urbano que contribuye a la orientación. }\end{array}$ \\
\hline $\begin{array}{l}\text { i.) } \\
\text { "Volver a la fachada continua" }\end{array}$ & $\begin{array}{l}\text { Este modelo de sustentabilidad que postula una mayor } \\
\text { densidad, resultará por sí mismo en el resurgimiento de la } \\
\text { fachada continua. Pero esta medida se postula aquí } \\
\text { vocativamente, a fin de promover el desplazamiento } \\
\text { peatonal, observando que los bordes edificados discontinuos } \\
\text { disuaden al peatón. } \\
\text { La percepción explica algunos motivos de esta preferencia, } \\
\text { que yendo más allá del reconocimiento que habitualmente se } \\
\text { hace del efecto de la presencia latente del control social que } \\
\text { ejercen edificios habitados que permean su actividad hacia la } \\
\text { calle, "dando sensación de compañía": } \\
\text { La fachada continua tiene alta comprensibilidad como un } \\
\text { apoyo que, percibido como límite, otorga la sensación de } \\
\text { amparo o de resguardo a imprevistos desde el flanco que } \\
\text { protege. Si en ella se distinguen constancias, ritmos y } \\
\text { proporciones que faciliten la lectura de claves para interpretar } \\
\text { la escala y el destino del edificio, se aumenta la atribución de } \\
\text { significados fiables, lo que disminuye el temor a lo } \\
\text { desconocido. Sin embargo si el ritmo es vertical y } \\
\text { excesivamente regular, la percepción de la fachada se hará }\end{array}$ \\
\hline
\end{tabular}


más larga de lo que efectivamente es (efecto de Helmholzt) $(\underline{36})$, disuadiendo al transeúnte a recorrerlo por la expectativa de 'lejanía' que produce. Y si dicha fachada mantiene su línea edificatoria constante y plana en la vertical, disuadirá al peatón por una percepción de no acogimiento, de rechazo, de indiferencia. Si por el contrario, las fachadas continuas presentan entrantes e inflexiones, se dice que favorecen el uso peatonal, al comunicar oportunidad de acogida y de discreta detención sin interrumpir el flujo. No obstante este mismo atributo positivo puede transformarse en negativo, si se perciben como caletas de antisociales. La fachada discontinua por su parte, si se ha generado por diversidad de la propiedad predial y libertad de empresa, será de baja comprensibilidad por falta de 'pregnancia' como un todo, y seguramente se presentará también baja en 'constancias'. Estas características la hacen muy desaconsejable para la formación de nociones y también para manejar mensajes de escala, ya que las cosas aisladas (ley de Rici) tienden a engañar al modificarse sus atributos, ante escasas referencia. Por su propio modelo, se suceden lapsos vacíos de actividad y consecuentemente de bajo control social y de bajo aliciente a recorrerlos por falta de estímulos.

\section{4.- Los Park Ways}

Tratamos esta tipología separadamente de las vías, ya que no se plantea como una más dentro de las medidas estratégicas del modelo de sostenibilidad, sino como un tipo que puede mantener sus características de 'parque recorrible' tanto al interior de una ciudad como también en extensiones rurales, ya que incluso su nacimiento obedeció al factor preceptivo, intentando un 'promenade', un viaje placentero.

Desde importantes variables de la visualidad se puede decir de ellos lo siguiente:

Por su propio concepto poseen - cada uno y el conjunto de ellos - 'unidad con variedad', que es uno de las leyes gestalt de la certera percepción.

Tienen al mismo tiempo el atributo de la 'totalidad' por lo ininterrumpido de una estructura clara, y por la nítida demarcación y diferenciación del resto del entorno. Su mismo significado es un total reconocible por todos, un parque.

Por ser vocativamente un parque reconocible, tiene 'carácter' e identidad, lo que junto con la 'totalidad' mencionada le confiere alta comprensibilidad.

La sucesión de elementos que, sin embargo son de naturaleza relativamente homogénea - vegetación y otros - que se desarrolla con infinitas variaciones a lo largo del corredor por ambos lados, hace que se perciba con claridad la 'profundidad' pero en una versión dinámica, debido principalmente a la atmósfera dado que aquí se favorecen las gradientes lumínicas al evitar los tramos excesivamente rectos que darían una perspectiva central y monótona, promotora de aceleración. 
El fundamental tópico perceptual de la discriminación entre figura / fondo, se ve facilitado en los Park Ways porque la figura que interesa a la conducción - la carpeta rodada- es claramente lo único artificial y racional en el paisaje del parque. Por su propio colorido, netamente claro recortada sobre oscuro, el fenómeno de discriminación figura/fondo, se favorece añadiendo sensación de seguridad.

No obstante estos atributos visuales positivos, sus flancos tratados sólo con vegetación y sin nada inesperado en las largas extensiones en que se aplica el modelo, hacen que surja prontamente un efecto 'entrópico' y se pierda la escala. A pesar de las curvas y sinuosidades que cualifican el total y muestran distintos enfoques panorámicos, de no mediar otros vehículos en el momento, la expectativa de distancia junto con la presencia de los ritmos verticales de la vegetación, hacen aumentar la longitud real.

\section{Conclusión}

Queda comprobada la contribución que pueden hacer las ciencias sociales, especialmente la rama de la sicología que se aboca a la percepción visual, puede ser de gran incidencia en el éxito de los postulados y en la estabilidad del modelo sobre movilidad urbana tendiente a la sostenibilidad. Considerar la PV como herramienta que pudiera aportar a la estabilidad y consolidación del modelo, desde individuos más conscientes de su espacio, con alto grado de empatía hacia su entorno a partir de una mayor comprensión de su ambiente, individuos orientados y habilitados desde allí para un mejor uso de su hábitat.

Como hemos demostrado, un entorno conductivo hacia una mayor cooperación, debe partir por ser un entorno fácilmente deductivo y capaz de ser inductivo a la vez. Ello sólo se logrará incrementando la cooperación multidisciplinal, para una mayor y más rigurosa consideración de factores que a veces aparecen a los estratos técnicos como meramente subjetivos y difícilmente aprehensibles, pero cuyo dominio y manejo está en la base de las decisiones específicas que aseguren el éxito del modelo.

Santiago de Chile, marzo 2002.

\section{Referencias}

\section{Bibliografía}

BANDLER Y GRINDLER, "Psicología y Percepción", Ed. Paidos, Barcelona, 1990.

BARTLEY, S. H., "Principios de Percepción", Ed. Trillas, Mx, 1980.

BURTON, H., "Going Green by Design". Urban Design Qualterly, Issue 57, january 1996.

CARTEReTte, E. Y FRIEDMAN, M., " Manual de Percepción", Ed. Trillas, Mexico, 1982. 
http://revistaurbanismo.uchile.cl

DEMATTEIS, G., "Suburbanización y periurbanización. Ciudades anglosajonas y ciudades latinas", Capítulo en 'La ciudad Dispersa' (S/e) (s/f).

EWING, R., "Is Los Angeles style sprawl desirable?", Journal of American Planning Association, V. 63 No 1, APA, Chicago, Il., Winter 1997.

GARRET, L., "Visual Design", Ed Van Nostrand Reinhold, Nueva York 1967.

GORDON, P. \& RICHARDSON, H.W., "Are Compact Cities a Desirable Planning Goal?" Journal of American Planning Association, V.63 No 1, APA, Chicago, Il., Winter 1997.

JURADO, D., "Psicografías Metro-politanas". Mapas Mentales y significación a partir del Metro de Santiago", Seminario Percepción y Significación, FAU, U. de Chile, 2001.

LETELIER, S., "Visualidad y Neguentropía. Equilibrio Visual", Ed. FAU, U de Chile, Santiago, 1993.

LURIA, A.R., "Sensación y Percepción", Ed Fontanella, Barcelona, 1981.

MERLEAU-PONTY, M., "Fenomenología de la Percepción", Ed. Península, Barcelona, 1997.

MONCLÚS, FCO. J. "Suburbanización y nuevas periferias. Perspectivas geográficourbanísticas". Capítulo en 'La ciudad Dispersa' (S/e) (s/f).

MUÑOZ, I., "I'm a Monument'. Percepción de la ciudad desde un Autobús", Seminario Percepción, 1996.

NORBERG-SCHULTZ, Ch., "Existencia, Espacio y Arquitectura", Ed. Blume, Barcelona, 1975.

POZUETA, J., "Planificación Urbanística y Transporte. Diseño de carreteras en áreas suburbanas", Separata rev. Ciudad y Territorio no 91-92, 1992., p.52.

POZUETA, J. "Movilidad y planeamiento urbanos sostenible". Cuadernos de Investigación Urbanística No 30, julio 2000.

ROZAS, S., "Eriazo, baldío, intersticial. Código de desarrollo espacial, generación y uso de vacíos en la periferia de Santiago" Seminario área Percepción / Significación, FAU. U de Chile. 2001.

SAUTHWORTH, M., "Walkable Suburbs? An Evolution of Neotraditional Communities at the Urban Edges. Journal of the American Planning Association Vol. 63 no 1, APA, Chicago, Il. Winter 1997. 


\section{REVSTADE URBANISMO}

http://revistaurbanismo.uchile.cl

VERNON, M.D., "Psicología de la Percepción" , Ed Paidos , Bs. As, 1967.

(S/A), "Compact, Decentralised or what? The Sustainable City Debate". No 2, (S/e) ( s/ f).

\section{Notas}

(1) S. H. Bartley, "Principios de Percepción", Ed Trillas, Mx, 1980.

(2) Gardner, cit M.D.Vernon, "Psicología de la Percepción" , Ed Paidos , Bs. As, 1967

(3) S.H Bartley, Op Cit, p.51-54

(4) A.R.Luria, Sensación y Percepción, Ed Fontanella, Barcelona, 1981

(ㅁ) M. Merleau-Ponty, "Fenomenología de la Percepción", Ed Península, Barcelona,1997.

(ㅁ) C. Norberg Schultz, "Existencia, Espacio y Arquitectura", Ed. Blume, Barcelona,1975

(7) E. Carterette/ M. Friedman, "Manual de Percepción", Ed Trillas, Mexico, 1982

() $\quad$ C. Norberg Schultz, Op. cit

(9) M. Merleau-Ponty, Op. cit.

(10) Psicología y Percepción. Bandler y Grindler, Ed Paidos, Barcelona, 1990

(11) Iván Muñoz 1996 y Diego Jurado 2001. Seminarios de Percepción y Sígnificación.

(12) POZUETA, J. 'Movilidad y planeamiento.... Cuadernos de Investigación Urbanística No 30, págs 33,34

(13) EWING 1997, cit POZUETA, Ibid., pág 37.

(14) Ibid, págs 60 a 64.

(15) Suburbanización y nuevas periferias. Perspectivas geográfico-urbanísticas. Fco. J. Monclús. Capítulo en 'La ciudad Dispersa' (S/e) (s/f)

(16) Compact, Decentralised or what? The Sustainable City Debate. No 2 ( S/e) ( s/f). p23 y 25

(17) Going Green by design. Hugh Burton Urban Design Qualterly, Issue 57, january 1996 . p5 y 6

(18) Compact, Decentralised or what? The sustainable city debate. No 2 (s/e), (s/f), p33-34

(19) Walkable Suburbs? An evolution of neotraditional communities at the urban edges. M. Sauth worth, Journal of the American Planning Association Vol63 no 1, Winter 1997, APA, Chicago, Il.. p41

(20) M. Sauth worth, Ibid, p34

(21) Hough cit. En Compact, Decentralised or what? (S/e) ....p2

(22) Hugh Burton, Op cit, p5

(23) Is Los Angeles style sprawl desirable? Reid Ewing, Journal of American Planning Association, V.63 No 1, Winter 1997 , APA, Chicago, Il.p.118

(24) Are compact cities a desirable planning goal? P. Gordon and H.W. Richardson. Journal of American Planning Association, V.63 No 1, Winter 1997 , APA, Chicago, Il., p99

(25) Reid Ewing, Op cit. p.108

(26) Suburbanización y periurbanización. Ciudades anglosajonas y ciudades latinas. Giuseppe Dematteis. Capítulo en 'La ciudad Dispersa' (S/e) (s/f), p.25-31.

(27) Dematteis Ibid, pág. 29.

(28) Compact, Decentralised or what? The sustainable city debate. No 2 (s/e), (s/f), p.31

(29) Eriazo, baldío, intersticial. Código de desarrollo espacial, generación y uso de vacíos en la periferia de Santiago. Sebastián Rozas, Seminario área Percepción / Significación, FAU. U de Chile. 2001

(30) Planificación Urbanística y Transporte. Diseño de carreteras en áreas suburbanas. Julio Pozueta, Separata rev. Ciudad y Territorio no $91-92$, 1992., p.52

(31) Ibid, p.54-55 


\section{REVSTADE

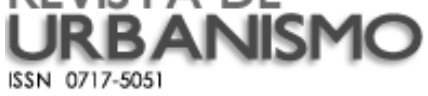

http://revistaurbanismo.vchile.cl

(32) Ibid., p.57-58

(33) J. Pozueta, Op.cit p48

(34) 'I'm a Monument' Percepción de la ciudad desde un Autobus, Iván Muñoz, Seminario Percepción, 1996

(35) Visualidad y Neguentropía. Equilibrio visual. Sofía Letelier, De FAU, U de Chile, Santiago, 1993.

(36) Cit. Lilian Garret, Visual Design, Ed. Van Nostrand Reinhold, Nueva York 1967

Nota de R.: Este escrito corresponde a un ejercicio realizado en el marco del Programa Doctoral de la Universidad Politécnica de Madrid. 